\title{
First onset of a left-bundle-branch ventricular tachycardia in an elderly: A rare cause
}

\author{
Katharina Kirsch ${ }^{1 *}$, Katharina Schöne ${ }^{2 *}$, Cosima Jahnke ${ }^{1,2}$, Ingo Paetsch ${ }^{1,2}$, \\ Astrid Monecke ${ }^{4}$, Andreas Schwarzer ${ }^{3}$, Holger Thiele ${ }^{1}$, Marcus Sandri ${ }^{1}$ \\ ${ }^{1}$ Department of Internal Medicine/Cardiology, Heart Center at Leipzig University, Leipzig, Germany \\ ${ }^{2}$ Department of Electrophysiology, Heart Center at Leipzig University, Leipzig, Germany \\ ${ }^{3}$ Practice Hematology, Oncology and Palliative Care, Leipzig, Germany \\ ${ }^{4}$ Institute of Pathology, University of Leipzig, Leipzig, Germany
}

A 78-year-old male patient was admitted for assessment of non-sustained left-bundle-branch ventricular tachycardia (VT) and recurrent syncope. According to the 12-lead electrocardiogram the morphologic origin was the right ventricular (RV) free wall (Fig. 1A). An arrhythmogenic RV cardiomyopathy or other structural cardiac conditions were not preexistent. By transthoracic echocardiography an abnormal thickness of the $\mathrm{RV}$ wall with preserved RV function accompanied by mild pericardial effusion was noted. Ischemic triggering of the VT was ruled out by coronary angiography. Cardiovascular magnetic resonance imaging revealed edematous and highly capillarized malignant infiltration of the entire RV myocardium (Fig. 1B, Suppl. Video 1).

The patient had a history of cutaneous tumor in his leg 1.5 years previous, which was diagnosed as T-cell non-Hodgkin lymphoma, also known as mycosis fungoides in the tumor stage. Since no further staging procedures including computed tomography scans were performed, a distinction between primary cutaneous T-non-Hodgkin lymphoma and peripheral T-non-Hodgkin lymphoma not otherwise specified (NOS) could not be made.

During hospital stay for VT no reliable diagnosis could be found by diagnostic pericardiocentesis with subsequent fluorescence-activated cell sorter analysis. Thus, a RV endomyocardial biopsy was taken. Analysis of biopsies of the free wall of the right ventricle showed an infiltration by an atypi- cally imposing lymphoid cell population associated with myocytic necrosis and extended expression of CD 3 T-cell marker (proliferation rate 80\%) (Fig. 1C). Immunohistochemistry for CD 30 showed a negative result. In summary, histology of RV biopsy showed an aggressive T-non-Hodgkin lymphoma (T-NHL NOS). Furthermore, a computed tomography scan revealed cerebral manifestation.

The following clinical course was dominated by recurrent VT including external cardioversion for termination. Unfavorable prognosis and treatment options with palliative chemotherapy were discussed with the patient. He decided for palliative care with amiodarone but no chemotherapy. One week subsequent to discharge he died while asleep.

Cardiac manifestations of lymphoma are rare and are mostly diagnosed by autopsy. Most reported cases of cardiac lymphoma have been of $\mathrm{B}$-cell origin and there are only a few cases of T-cell-lymphoma with intrapericardial localization. There is a large diversity in clinical manifestations, including arrhythmias, chest pain and heart failure.

A characteristic feature of cardiac T-cell lymphoma is an intrapericardial growth and lymphatic spread with infiltration of the complete myocardium. This case highlights that a first onset of left-bundle branch VT in the elderly may be caused by a rare cardiac manifestation of T-non-Hodgkin lymphoma.

Conflict of interest: None declared

\footnotetext{
Address for correspondence: Marcus Sandri, MD, Heart Center Leipzig - University Hospital, Department of Internal Medicine/Cardiology, Strümpellstraße 39, 04289 Leipzig, Germany, tel: +49 341865 1428, fax: +49 3418651461 , e-mail: marcus.sandri@medizin.uni-leipzig.de

Received: 05.08.2018 Accepted: 12.10.2018

*Katharina Kirsch and Katharina Schöne contributed equally.
} 


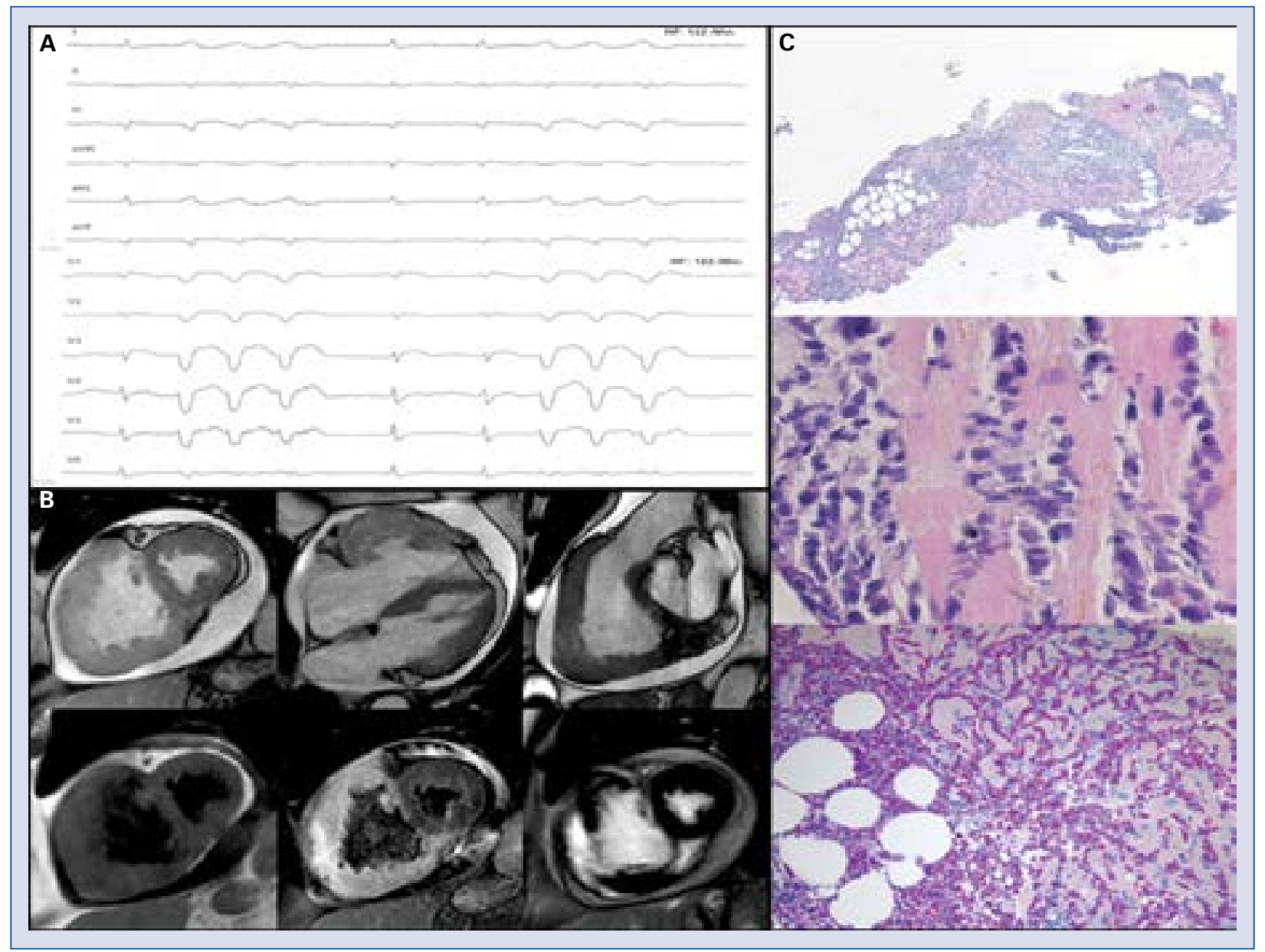

Figure 1. A. Electrocardiogram (ECG). Twelve-lead ECG tracing of left-bundle-branch ventricular tachycardia with the ECG morphology being suggestive of a right ventricular (RV) free wall origin. B. Cardiovascular magnetic resonance functional imaging and tissue characterization. Upper panel: Cine imaging demonstrated severe thickening of almost the entire RV wall on short axis, 4-chamber, and RV outflow tract geometry and an accompanying circular pericardial effusion with diastolic impression of the right-atrial wall was seen. Lower panel: Myocardial tissue characterization of the RV wall (short axis orientation). While T1-weighted images signal intensity of the RV mass was identical to left ventricular myocardium serving as reference tissue, the homogeneously hyperintense signal intensity on T2-weighted images revealed a highly capillarized/edematous composition of the mass with localized areas of positive late enhancement found mainly in the RV free wall. In addition, the signal intensity increases in the entire mass during the first-pass, contrast-enhanced perfusion imaging proved its coronary arterial supply representing a clear indicator of malignancy. Thus, cardiac magnetic resonance myocardial tissue characterization promptly diagnozed the malignant infiltration of RV myocardium. C. Histopathology of RV myocardium. Upper panel: Biopsy sample (5-fold magnification, hematoxylin-eosin staining): small-sized necrosis in myocardium infiltrated by atypical lymphocytes. Mid panel: Myocardium and lymphomatous tissue (100-fold magnification, hematoxylin-eosin staining): preserved cross striation of cardiomyocytes, lost myocardial striation and cell edema with fuzzy cell boundaries as a sign of necrosis. Lower panel: Staining for T-cell marker CD 3 identifying an atypical T-cell population (20-fold magnification). 